\title{
Deep minima in stellar dynamos
}

\author{
J. Brooke ${ }^{1,2}$, D. Moss ${ }^{2}$, and A. Phillips ${ }^{2, \star}$ \\ ${ }^{1}$ SVE Group, Manchester Computing, University of Manchester, Manchester M13 9PL, UK \\ 2 Department of Mathematics, University of Manchester, Manchester M13 9PL, UK
}

Received 9 July 2002 / Accepted 10 September 2002

\begin{abstract}
We study a Cartesian analogy for a solar dynamo model to investigate systematically a dynamo model limited by the back reaction of the Lorentz force on the differential rotation. In particular, we investigate intermittent behaviour found at low turbulent magnetic Prandtl numbers $\tau$, and determine empirical scaling laws with $\tau$. We find this class of models to be incapable of producing extended periods of "normal" behaviour separated by occasional "grand minima" - rather the behaviour is a mirror image, with occasional "grand maxima". Further we find the existence of solar-like torsional oscillations to be incompatible with low magnetic Prandtl number intermittent regimes.
\end{abstract}

Key words. magnetic fields - magnetohydrodynamics (MHD) - Sun: magnetic fields

\section{Introduction}

In this paper we investigate the dynamics of deep minima in stellar and solar dynamo models. The term deep or grand minima describes intervals of abnormally low magnetic activity which persist for a number of activity cycles, this distinguishing them from the minima that occur during each magnetic activity cycle (Eddy 1978; Ribes \& Nesmes-Ribes 1993). From proxy records, such as deposition of radio-isotopes, such deep minima in solar activity are inferred to have occurred at irregular intervals over the last 10000 years, with a characteristic average interval of 200 years (i.e. about 18-19 Schwabe cycles). Thus there is a suggestion of a characteristic timescale of these deep minima. Evidence also exists that similar dramatic changes in activity levels occur in other solar-like stars, both from observations that about $30 \%$ of the stars that might be expected to be chromospherically active at a given time are not (White et al. 1992), and from observations of sudden changes in activity levels of a few stars (Baliunas et al. 1995).

Recently, different dynamo models have reproduced the phenomena of anomalously deep low states, either by stochastic fluctations (Schmitt et al. 1996) or deterministically through different forms of chaotic behaviour. The deterministic models show two broad classes of mechanism which can be identified with behaviour exhibited in the theory of bifurcations of loworder dynamical systems.

One such behaviour is intermittency, where there is a switching between different states with mechanisms that evolve

Send offprint requests to: J. Brooke,

e-mail: j.m.brooke@mcc.ac.uk

* Current address: NASA Goddard Space Flight Center, Code 931, Greenbelt MD 20771, USA. one state into another. In the solar context we would identify one state with solar activity as observed from the mid-17th century and the other state with the abnormally low activity of the Maunder minimum. The proxy records indicate switching between these states at irregular intervals over the entire span of records. This switching was interpreted as "on-off" intermittency by Platt et al. (1993a,b) and related to the dynamics of the solar grand minima. In its original form this required that the dynamo inferred to be operating at the base of the solar convection zone be close to the bifurcation from the critical state, and the noise provided by the convection zone above it alternately switched it above and below the bifurcation point. Subsequent work has shown that this assumption of near-criticality is not necessary. A more general mechanism termed as "in-out" or "icicle" form of intermittency was identified by Brooke et al. (1998) and Ashwin et al. (1999). Here the "out" phase describes a leisurely evolution where the characteristics of the cycle remain apparently unchanged until there is a sudden crisis in the system. This is followed by a restoration of the previous behaviour (the "in" phase). Such behaviour is intimately connected with the breaking of equatorial symmetry (Brooke 1997), and is motivated by the evidence that the solar cycle was highly asymmetric about the equator during and just after the Maunder Minimum (Sokoloff \& Nesme-Ribes 1994).

Another interesting deterministic mechanism is aperiodic modulation of the basic Schwabe cycle in a system that has two distinct timescales. This was first described in low-order models (Weiss et al. 1984), but has also been demonstrated by Tobias (1996b, 1997) in a numerical model of an interface dynamo with two degrees of spatial freedom. Here the idea is that the magnetic (Schwabe) cycle and the modulation have different timescales whose ratio is, for example, a function of the 
turbulent magnetic Prandtl number $\tau=v / \eta$, where $v$ and $\eta$ are the turbulent viscosity and magnetic diffusivity respectively (see next section for details). The physics of this mechanism is that in the absence of magnetic field, there is a zeroth order rotation law (maintained by means such as turbulent Reynolds stresses (e.g. Rüdiger 1989) whose profile is such that it can support the growth of a seed magnetic field via dynamo action. As this field becomes sufficiently large it alters the zeroth order velocity field via the Lorentz force (the Malkus-Proctor mechanism, Malkus \& Proctor 1975) to a configuration where the dynamo action is no longer supported. The field then decays on the ohmic decay timescale until the Lorentz force is sufficiently weak that the velocity field can diffuse back to the original zeroth order form on the viscous timescale, at which time the growth phase starts again. For small values of $\tau$ we can clearly obtain a long timescale modulation of the magnetic cycle, and Tobias (1996) suggested that the long timescale scales as $\tau^{-1 / 2}$, although the range of $\tau$ that was accessible to him numerically was rather limited.

In this paper we wish to explore this mechanism further by exploring behaviour over a wide range of magnetic Prandtl numbers and looking for scaling behaviour. Additionally, we comment on recent work that has shown that the Lorentz force can also drive the torsional oscillations, that is waves of variation in the solar rotational velocity that appear to track the migration of the low latitude magnetic field belts (Howard \& LaBonte 1980). We seek to determine whether there are ranges of magnetic Prandtl number where the Lorentz force can act on both the magnetic and kinematic scales simultaneously. In this context, we note that there is some evidence that the equatorial solar rotation rate as determined from sunspot observations was slower in the period of the Maunder minimum when the magnetic field was abnormally weak (Ribes \& Nesme-Ribes 1993). Since the torsional oscillations also relate to variations in the differential rotation, this suggests the possibility that the same physical mechanism may be operating in both phenomena but on differing time-scales.

Moss \& Brooke (2000) made a preliminary investigation of low magnetic Prandtl number regimes in a spherical model with an underlying rotation law based on helioseismological inversions. Phillips et al. (2002, hereafter Paper I) also investigated a Cartesian model, over a range of parameters. At small magnetic Prandtl numbers, Moss \& Brooke's results were sufficiently similar in broad dynamical properties to the Cartesian box solutions to warrant a more thorough investigation of this regime in the simplified geometry (note that this correspondence does not extend in detail to magnetic Prandtl numbers of about $10^{-1}$ or larger).

Such an investigation is numerically challenging. Computations with either $0<\tau \ll 1$ or $\tau \gg 1$ require extremely long integration times to resolve long term behaviour on both of the timescales present. Thus, until recently, investigations generally have been conducted for kinematic models where $\tau$ does not feature in the dynamo equations (e.g. alpha-quenched models), or for dynamical models with magnetic Prandtl numbers of order unity. It is an open question whether the turbulent magnetic Prandtl number can deviate significantly from unity. We take $\tau$ to be a free parameter, in order to see whether any range of values can give solar-like behaviour. We also find some results for small $\tau$ that are of more abstract interest in dynamo theory.

In this paper we revert to the Cartesian model with purely radial rotational shear used by Tobias $(1996,1997)$ despite the fact that several of its features in terms of boundary conditions, rotation law and ratio between the length scales in the overshoot and convective regions do not adequately represent the Sun. We have elsewhere made an extended appraisal of variants of this model as more and more solar-like features are added (Phillips 2001, Paper I). Our main aim here is to isolate the effects of varying $\tau$ in a controlled manner.

\section{The numerical model}

\subsection{Derivation of equations}

We work in a Cartesian co-ordinate system $(x, y, z)$, where the latitude is represented by the co-ordinate $x, 0 \leq x \leq 2 L$, with the north (south) pole at $x=0(x=2 L)$ and the equator at $x=L$. The co-ordinate $z, z_{l} \leq z \leq 1$ corresponds to the radial direction, with the overshoot layer $\left(z_{l} \leq z<0\right)$ and convection zone $(0<z \leq 1)$ separated by an "interface" at $z=0$. Since the unit vector $\hat{\boldsymbol{x}}$ points tangentially south along the meridian, with $\hat{z}$ radially outwards, $\hat{\boldsymbol{y}}$ is directed eastwards in the direction of the rotational velocities. The assumption $\partial / \partial y \equiv 0$ thus corresponds to imposing axisymmetry.

The magnetic field may be written as $\boldsymbol{B}=B \hat{\boldsymbol{y}}+\nabla \times(A \hat{\boldsymbol{y}})$, where $A(x, z, t)$ and $B(x, z, t)$ are scalar functions corresponding to the poloidal vector potential and toroidal field. Thus, $\nabla \cdot \boldsymbol{B}=0$ automatically. The velocity field can be expressed as $\boldsymbol{V}=V \hat{\boldsymbol{y}}=(U+v) \hat{\boldsymbol{y}}$, where we split the scalar function $V(x, z, t)$ into two parts, $U(x, z)$ and $v(x, z, t)$. The first of these corresponds to the (time averaged) rotation law (in this paper with purely radial shear but it could, for example, be inferred from helioseismology), whereas the second component represents perturbations to the zero-order velocity state driven by Lorentz forces, which act to modify differential rotation and quench the $\omega$-effect. This is the sole nonlinearity used in this paper.

In the $\alpha \omega$ limit, differential rotation is assumed to be solely responsible for the generation of toroidal field from poloidal. (Selected computations using an $\alpha^{2} \omega$ model suggest this approximation is valid for all of the cases presented in this paper). From the definition of the model, it is clear that the system should return to the zero-order state in the absence of a magnetic field. We solve the mean field dynamo equation for $A(x, z, t), B(x, z, t)$, and the $y$-component of the momentum equation for $v(x, z, t)$. These are non-dimensionalized by setting

$x=l \tilde{x}, \quad z=l \tilde{z}, \quad t=l^{2} \tilde{t} / \eta_{0}, \quad A=A_{0} \tilde{A}, \quad B=B_{0} \tilde{B}$,

$U=l \Omega_{0} \tilde{U}, v=l \Omega_{0} \tilde{v}, \quad \alpha=\alpha_{0} \tilde{\alpha}, \quad \eta=\eta_{0} \tilde{\eta}$,

$\nu=v_{0} \tilde{v}, \quad \rho=\rho_{0} \tilde{\rho}$,

where $l$ is a characteristic length scale in the $z$-direction (corresponding to the convection zone depth), and $\alpha_{0}, \Omega_{0}, \eta_{0}, v_{0}$ and $\rho_{0}$ are typical values of the $\alpha$, angular velocity, magnetic diffusivity, viscosity and density. 
Given that we are studying an $\alpha \omega$ dynamo, defining $A_{0} / B_{0}=\alpha_{0} l^{2} / \eta_{0}$ explicitly demonstrates the existence of the single dynamo number $D$. Assuming the density to be constant and omitting the tildes, we obtain

$\frac{\partial A}{\partial t}=\eta \nabla^{2} A+\alpha B$,

$\frac{\partial B}{\partial t}=\eta \nabla^{2} B+D\left[\frac{\partial A}{\partial x}\left(\frac{\partial U}{\partial z}+\frac{\partial v}{\partial z}\right)-\frac{\partial A}{\partial z}\left(\frac{\partial U}{\partial x}+\frac{\partial v}{\partial x}\right)\right]$,

and

$\frac{\partial v}{\partial t}=\tau\left[\eta \nabla^{2} v\right]+\operatorname{sgn}(D)\left[\frac{\partial A}{\partial x} \frac{\partial B}{\partial z}-\frac{\partial A}{\partial z} \frac{\partial B}{\partial x}\right]$.

We have ignored any meridional circulation in deriving Eq. (3), and the value of $B_{0}$ has been chosen so that the nonlinear terms in Eq. (3) are multiplied by a coefficient of magnitude unity. The controlling parameters are

$D=\frac{\alpha_{0} \Omega_{0} l^{3}}{\eta_{0}^{2}}, \quad \tau=\frac{\nu_{0}}{\eta_{0}}$,

where $D$ is the dynamo number and $\tau$ is the magnetic Prandtl number.

Our modelling is motivated by the interface model of Parker (1993), but numerical considerations require that the radial profiles of the $\alpha, \omega$ and magnetic diffusivity coefficients are continuous. We follow Tobias (1997) and define the $\alpha$-effect by

$\alpha=f(z) \cos \left(\frac{\pi x}{2 L}\right)$,

where

$f(z)= \begin{cases}0, & z \leq-0.75, \\ \frac{1}{2}[1+\tanh 5 z]\left[1-\mathrm{e}^{-25(z+0.75)^{2}}\right], & z>-0.75 .\end{cases}$

Similarly, the zero-order velocity is defined as

$U(x, z)=u(z) \sin \left(\frac{\pi x}{2 L}\right)$.

Thus, the basic angular velocity shear is taken to be strictly radial, whilst a sine term has been included to mimic the effects of curvature, ensuring that $U=0$ at the poles (cf. Jennings \& Weiss 1991). $g(z)=\mathrm{d} u / \mathrm{d} z$, where

$g(z)=\left\{\begin{array}{lr}\frac{1}{2}[1-\tanh 5(z-0.5)]\left[1-\mathrm{e}^{-25(z-0.75)^{2}}\right], \\ 0, & z<0.75, \\ & z \geq 0.75,\end{array}\right.$

(Tovias 1996). We also assume that $z_{l}=-1$, and that $\eta=$ const, although other assumptions are certainly possible (e.g. Paper I). The functions $f(z)$ and $g(z)$ are displayed in Fig. 1 of Paper I; $f(z)$ increases from zero for $z \leq-0.75$ to close to unity for $z \gtrsim 0.5$, whereas $g(z)=+1$ for $z \leq 0$ and decreases to zero as $z \rightarrow 1$.

We define the three quantities

$E_{\mathrm{m}}=\frac{1}{V} \iint_{V} B^{2} \mathrm{~d} V, \quad E_{\mathrm{k}}=\frac{1}{V} \iint_{V} v^{2} \mathrm{~d} V$,

$P=\frac{E_{\mathrm{q}}-E_{\mathrm{d}}}{E_{\mathrm{q}}+E_{\mathrm{d}}}$.
Thus, $E_{\mathrm{m}}$ and $E_{\mathrm{k}}$ are energies associated with the toroidal magnetic field and the kinetic energy of the velocity perturbations, averaged over the spatial grid. $E_{\mathrm{q}}$ and $E_{\mathrm{d}}$ are the energies associated with the symmetric and antisymmetric parts of the toroidal field, and $P$ is the field parity: for a purely quadrupolar/dipolar field, $P=+1 /-1$.

\subsection{Boundary conditions}

We take as boundary conditions

$A=B=v=0, \quad x=0,2 L$,

(i.e. on the "polar" boundaries),

$A=B=v=0, \quad z=-1$,

and

$\frac{\partial A}{\partial z}=B=\frac{\partial v}{\partial z}=0, \quad z=1$.

\section{Results}

\section{1. $\tau=10^{-3}$, trends with dynamo number}

Our aim is to bring into clear focus the role of $\tau$ in causing deep minima. We can isolate the dynamics of widely separated magnetic and kinematic diffusive time scales by considering the behaviour as $\tau \rightarrow 0$. We begin by examining solutions with $\tau=10^{-3}$, shown in Fig. 1 . We start with $D=-300$, where linear analysis shows that only a dipole mode is excited at this value. We then increase $|D|$.

At this small magnetic Prandtl number the time series of the global magnetic energy $E_{\mathrm{m}}(t)$ for $D=-300$ (shown in Fig. 1a) no longer resembles the singly periodic, dipolar solutions obtained with $5 \times 10^{-3} \leq \tau \leq 10$. The magnetic energy is now modulated on two timescales: a short period of $O(1)$ (not visible on the scale of Fig. 1), and a longer period linked with variations in the kinetic energy. The behaviour is reminiscent of that presented in Fig. 10a of Moss \& Brooke (2000), except that it is far more regular and there are no departures from the purely antisymmetric state. Fourier analysis of the time series confirms the existence of two periods, with lengths $T_{1} \approx 0.63$ and $T_{2} \approx 315$. The approximate $1: 500$ ratio (cf. $\tau^{-1}=1000$ ) suggests that the Malkus-Proctor effect is responsible for the long-term fluctuations in $E_{\mathrm{m}}$ and the occurancies of grand minima. This is an example of the Type II modulation described by Knobloch \& Landsberg (1996) which is characterised by large variations in energy and comparitively small change in the parity.

\subsection{Variations in parity}

The values of the critical dipolar and quadrupolar dynamo numbers for this model are $D_{\mathrm{c}}(D) \approx-264, D_{\mathrm{c}}(Q) \approx-310$, and so it is unsurprising that the solution obtained with $D=-300$ is purely dipolar. It has been demonstrated that interactions between symmetric and antisymmetric field components can 

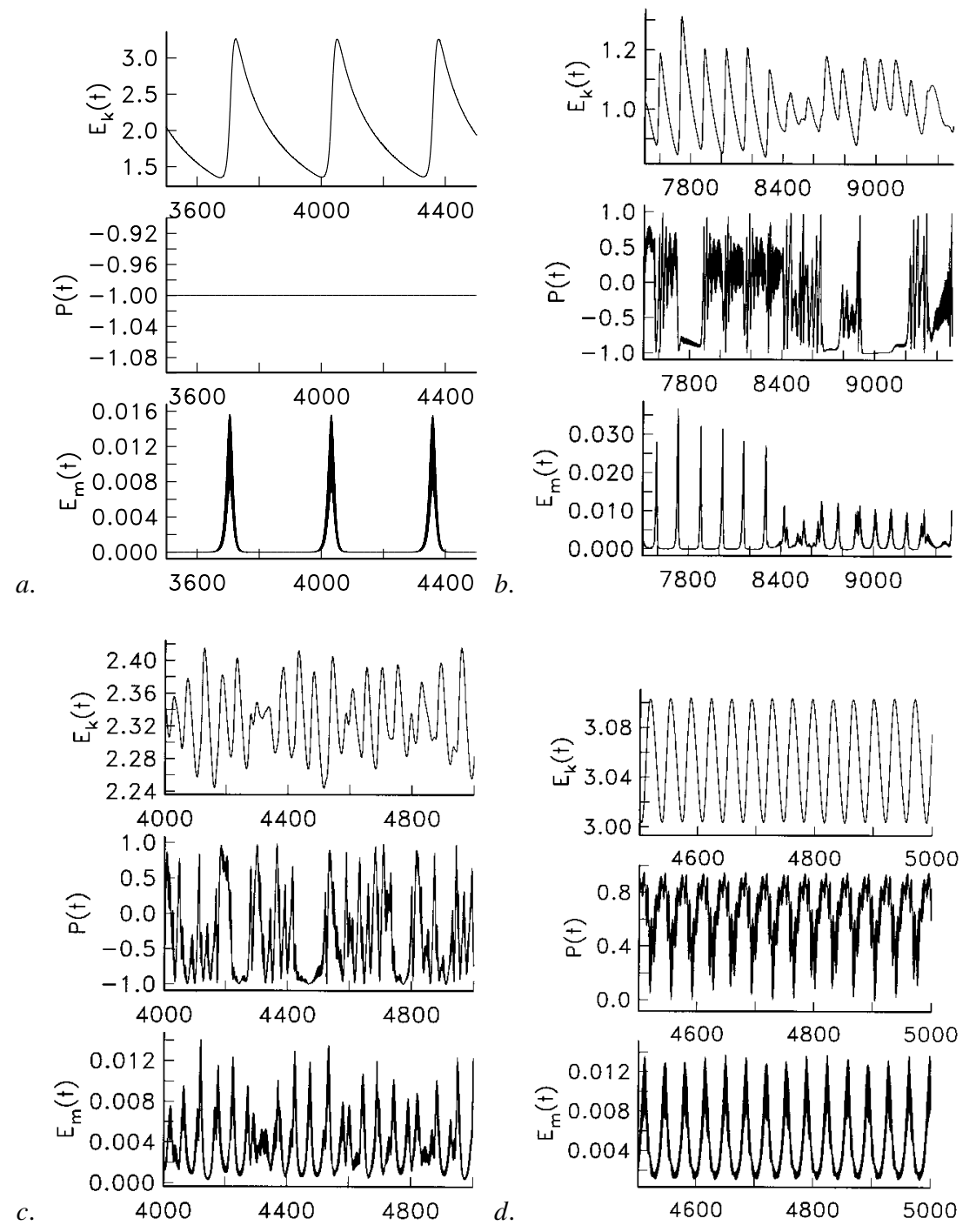

Fig. 1. Time series of magnetic energy $E_{\mathrm{m}}$, parity $P$ and perturbation kinetic energy $E_{\mathrm{k}}$ for $\tau=10^{-3}$ and a) $D=-300$, b) $D=-400$, c) $D=-600$, d) $D=-800$. The horizontal axes are labelled with values of dimensionless time, $t$.

lead to periods of reduced magnetic activity (Knobloch \& Landsberg 1996; Brooke et al. 1998). This suggests that performing computations with $|D| \gtrsim 310$ (where both dipolar and quadrupolar modes are excited) could result in grand minima coinciding with departures from the $P=-1$ state.

Figure $1 \mathrm{~b}$ shows a typical portion of the time series computed with $D=-400$. As expected, the behaviour is more complicated than that observed previously, with variations in parity as well as in magnetic and kinetic energy. This particular segment $(7500 \leq t \leq 9500)$ has been chosen since it is illustrative of the overall long-term behaviour. Fourier analysis of the entire computed $E_{\mathrm{m}}$ time series $(500 \leq t \leq 13000)$ reveals two broad peaks, corresponding to periods of $T_{1} \approx 0.57$ and $T_{2} \approx 130$. Similar estimates (within $10 \%$ ) were obtained from Fourier analysis of individual time series segments of length $\Delta t=2000$, indicating no significant change in the periods of oscillation. From inspection of Fig. 1b, $T_{1}$ clearly corresponds to the dynamo period, whilst $T_{2}$ may be identified as the average time separating the long term "bursts" in magnetic activity.
An interesting feature of this time series is that it switches between two different models of behaviour. In one, spikes in magnetic energy occur at quite regular intervals $(t=$ $7500-8300$ ); this behaviour is similar to that at $D=-300$. In the other, the spikes in magnetic energy are smaller in magnitude and less regular. Alternation of these two types of behaviour were found to occur throughout the entire computed time series $(500 \leq t \leq 13000)$. This switching may be an indication that we are here at the boundary between two different types of dynamical behaviour and the switching is an intermittent process.

Increasing $|D|$ results in a faster growth of the magnetic field when the dynamo is turned back "on", and hence shorter episodes of grand minima. Figures $1 \mathrm{c}, \mathrm{d}$ show the time series computed with $D=-600$ and -800 , supporting this conjecture. Although the overall pattern of behaviour is reminiscent of that for $D=-400$, the time-averaged kinetic energy $\left\langle E_{\mathrm{k}}\right\rangle$ increases as $\tau$ decreases. At $D=-600$ the long-term modulations in magnetic energy occur on a much shorter timescale, with relatively few instances where $E_{\mathrm{m}} \approx 0$. Furthermore, the 
maximum energy varies substantially from one cycle to the next, and there are no sections of the time series where the behaviour could be considered regular. When $D=-800$, the behaviour is much more regular. This re-establishment of periodic behaviour at higher values of the bifurcation parameter compared with chaotic solutions has been found in other dynamo models, as well as models showing the Ruelle-Takens route to chaos via bifurcation from solutions on a torus.

As expected, there are no prolonged episodes where $E_{\mathrm{m}} \approx 0$, when $|D| \gtrsim 400$ Although solutions have only been determined for a limited selection of parameters, the time series computed with $\tau=10^{-3}$ are unlike any of those found with $\tau=0.1$.

\subsection{Investigation of scalings with $\tau$}

To investigate scaling properties of the solutions, computations were performed at magnetic Prandtl numbers in the range $10^{-4} \leq \tau \leq 10$, for dynamo numbers $D=-300$ and $D=-400$. The behaviour was found to depend on the value of $D$, but we were only able to investigate systematically a very limited set of values. When $D=-300$, the solutions were found to be strictly periodic (singly for larger values of $\tau$, doubly for $\tau$ sufficiently small), with pure dipolar parity, whereas when $D=-400$ the solutions were of mixed parity and for smaller values of $\tau$ displayed irregular behaviour with time.

For $\tau \leq 10^{-2}$, the form of these solutions is similar to those shown in Figs. 1a,b, with the ratio of periods between the deep minima and the magnetic oscillations varying with $\tau$. As the magnetic Prandtl number increases the amplitude and period of the modulation of the magnetic oscillations both decrease. Thus the phenomenon of the extended deep minima gradually disappears.

When $D=-300$, we find that $\left\langle E_{\mathrm{m}}\right\rangle \propto \tau$ and, for $\tau \lesssim 10^{-3}$, $T \propto \tau^{-1}$. With $D=-400$, both dipolar and quadrupolar modes are excited in linear theory, and the nonlinear behaviour is considerably more complex (cf. Figs. 1a,b) when $\tau \lesssim 10^{-2}$. There is still a linear relationship between $\log \left\langle E_{\mathrm{m}}\right\rangle$ and $\log \tau$, indicating a power law variation of the form

$\left\langle E_{\mathrm{m}}\right\rangle \propto \tau$,

thus $\langle B\rangle \underset{\sim}{\propto} \tau^{1 / 2}$. Also, defining the mean period between grand maxima to be $\langle T\rangle$, and the mean energy of these maxima to be $<E_{\max }>$, we find

$\langle T\rangle \propto \tau^{-2 / 3}$,

and

$\left\langle E_{\max }\right\rangle \stackrel{\sim}{\sim} \tau^{2 / 3}$.

\subsection{The limiting case $\tau=0$}

Although performing computations with magnetic Prandtl numbers of $0<\tau \ll 1$ is computationally very expensive, it is feasible to investigate the behaviour when there is no viscous diffusion, i.e. $\tau=0$. In this limit, the evolution of $\partial v / \partial t$ is solely influenced by the actions of the Lorentz force (see Eq. (3)).

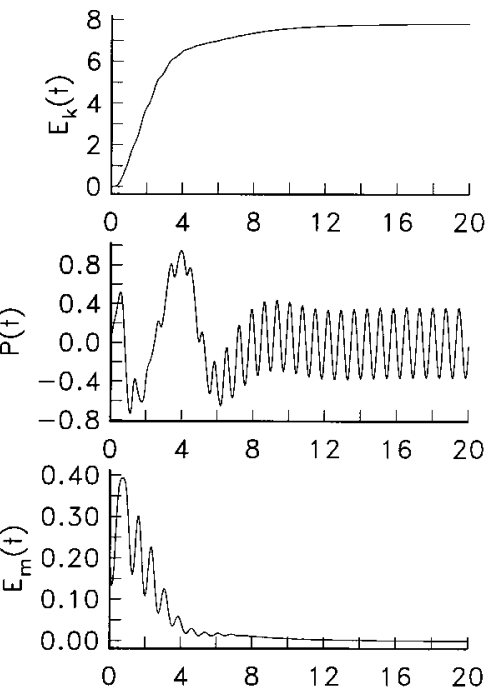

Fig. 2. As for Fig. 1, except with $\tau=0$. As $E_{\mathrm{m}} \rightarrow 0$, the velocity perturbations become "frozen" in space and time, with $E_{\mathrm{k}}$ tending to a constant, non-zero value.

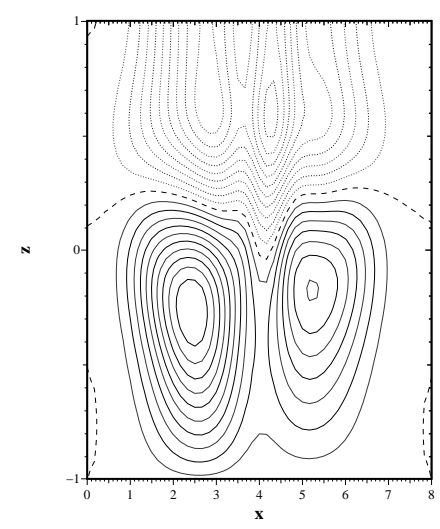

(a)

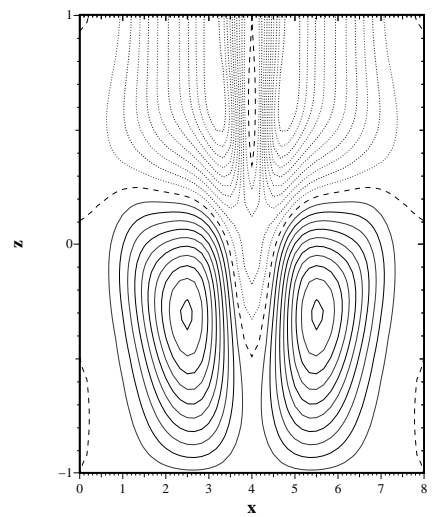

(b)
Fig. 3. Contours of perturbation velocity $v(x, z)$, computed with $D=-300, \tau=0$ and initial conditions a) $B(0)=B_{0}, P(0) \approx 0$, b) $B(0)=B_{0}, P(0)=-1$. Snapshots were taken at $t=20$, after which changes to the velocity field were assumed to be negligible. Clearly, the final solution state is not unique.

Then, once the Malkus-Proctor effect has altered the differential rotation so that dynamo action is no longer possible, there is no mechanism to restore the velocity to the zero-order state (see also Sect. 4.1). The magnetic field will thus decay indefinitely, as in the "self-killing" dynamo mechanisms described by Brummel et al. (1998) and Fuchs et al. (1999). This should be true even for $|D| \gg\left|D_{\mathrm{c}}\right|$. Linear analysis using the resulting (perturbed plus steady) velocity field $v$ should then confirm that $|D|<\left|D_{\mathrm{c}}^{*}\right|$, where $D_{\mathrm{c}}^{*}$ is the critical dynamo number with the frozen in perturbations to the basic velocity field.

In order to test these hypotheses we performed calculations with $\tau=0, D=-300$ and initial parity $P(0) \approx 0$. The time series for this case is shown in Fig. 2. There is a brief period of growth in magnetic (and kinetic) energy, followed by 
a sharp, exponential decline of the magnetic field with oscillations in parity. The onset of decay is rapid, with $E_{\mathrm{m}}$ becoming negligibly small after the elapse of only a few ohmic diffusion times $(t \approx 5)$. As $E_{\mathrm{m}} \rightarrow 0, \partial v / \partial t \rightarrow 0$ and $E_{\mathrm{k}}$ tends to a constant value. A contour plot of the resulting perturbation velocity field $v(x, z)$ is shown in Fig. 3a. Linear computations with the eventual total velocity field (i.e. zero-order plus perturbation) gave estimates for the critical dipolar and quadrupolar dynamo numbers of $D_{\mathrm{c}}^{*}(D) \approx D_{\mathrm{c}}^{*}(Q) \approx-347$. Thus the Lorentz force feedback has led to an increase in the effective value of $\left|D_{\mathrm{c}}\right|$. Computations with larger values of $|D|$ also lead to decaying magnetic fields, although it takes longer for dynamo action to be quenched. This is to be expected, since "killing" more supercritical dynamos requires larger perturbations to the zero-order velocity.

The velocity contours shown in Fig. 3a are clearly asymmetric about the equator $(x=4)$. Since the parity at $t=0$ was defined to be $P(0) \approx 0$, this suggests that the final velocity state may depend on the initial magnetic field. Computations with $P(0)=-1$ and $P(0)=+1$ (and $D=-300$ ) lead to qualitatively similar behaviour with time of $E_{\mathrm{m}}$ and $E_{\mathrm{k}}$, but the corresponding contours of $v(x, z)$ are both purely symmetric, although not identical. Also, for example, beginning the computation with $P(0) \approx 0$ again, but with the seed magnetic field uniformly increased in strength by a factor of 10 produces yet another (asymmetric) final velocity distribution. Thus, the the final solution state is not unique (see e.g. Figs. 3a,b).

\section{Analysis}

\subsection{Physical basis of the observed dynamics}

The long-term pattern of magnetic energy growth and decay may be explained by considering the evolution of the $\boldsymbol{B}$ and $\boldsymbol{V}$ fields in the limit of small $\tau$. Consider an instant in time at which $E_{\mathrm{m}}$ is small and the velocity field is only weakly perturbed from its zero-order state. Then, assuming $|D|>\left|D_{\mathrm{c}}\right|$, the magnetic field (and consequently $E_{\mathrm{m}}$ ) grows as a result of dynamo activity. This leads to stronger Lorentz feedback, and the velocity perturbations (measured by $E_{\mathrm{k}}$ ) rapidly increase in magnitude. Ultimately, the $\omega$-effect is sufficiently reduced that dynamo action is no longer sustainable, and the magnetic field begins to decay exponentially. There follows a corresponding reduction in the Lorentz force, allowing the velocity field to diffuse back to its initial, zero-order state. Since $\tau \ll 1$, ohmic dissipation occurs on a much shorter timescale than viscous diffusion. Thus the behaviour is very aperiodic, with extended minima for smaller values of the dynamo number. Once the velocity perturbations become sufficiently reduced, the dynamo is no longer quenched, the magnetic field begins to grow again, and the process repeats.

In a simple minded picture, the field will grow to about the same maximum strength (for given $D$ ) before the velocity field is sufficiently perturbed to affect the dynamo (essentially when the terms on the right hand side of Eq. (3) come into balance). After the decay of the magnetic field on the ohmic timescale $(O(1))$, the it remains in a low state until the velocity perturbations decay on the viscous timescale $\left(O\left(\tau^{-1}\right)\right.$. This picture appears to be consistent with the low magnetic Prandtl number behaviour at $\tau \lesssim 10^{-3}$ when $D=-300$, where $E_{\max } \sim$ const and $T \propto \tau^{-1}$ (Sect. 3.3). However the situation is clearly less simple when $D=-400$, see relations (6), (7), (8). Plausibly this is associated with the symmetry breaking present in these solutions or, more fundamentally, with the velocity field perturbations that quench one dynamo mode not necessarily being of the same form as those that quench the other parity mode.

The two phases of behaviour (growth and decay) are similar in character to the "self-killing" dynamos described by Brummell et al. (1998) and Fuchs et al. (1999), where dynamo action initially occurs, but the velocity field is subsequently distorted so that dynamo action is not possible, and the magnetic field thus eventually decays. Extensive computations with magnetic Prandtl numbers much smaller than $\tau \sim 10^{-3}$ are impractical, due to the long integration times required. However, provided $\tau>0$ and that the dynamo is supercritical in the zeroorder state $(v(x, z)=0)$, it can be seen that $E_{\mathrm{m}}$ will never decay indefinitely, but that the "low" state will persist for relatively longer intervals.

\subsection{Chaotic oscillations in parity}

In the context of an alpha-quenching mechanism, another possible mechanism for producing grand minima was proposed by Brooke et al. (1998). There a steadily growing quadrupolar component of magnetic field interacts with a (much stronger) dipolar component, resulting in an overall dip in magnetic energy. This behaviour fits the description of in-out intermittency (Ashwin et al. 1999; Covas et al. 2001a), a generalisation of onoff intermittency (Platt et al. 1993). The "in-out" terminology refers to two phases of activity: the "out" phase where the parity moves slowly away from $P=-1$, and the "in" phase characterised by a drop in magnetic energy and a return to almost pure dipolar parity. Here, the time interval between grand minima is determined by the length of the "out" phase, as opposed to the value of the magnetic Prandtl number in the discussion above. We can examine the equatorial symmetry breaking shown in the time series of Fig. $1 \mathrm{~b}$ to see if there are episodes where there is an interchange of energy between the symmetric and antisymmetric components of the magnetic field, one of which is steadily growing as in the "out" phase described above. We decompose the magnetic energy $E_{\mathrm{m}}$ into its dipolar and quadrupolar components, $E_{\mathrm{d}}$ and $E_{\mathrm{q}}$, and (somewhat arbitrarily) define

$C=\frac{2 \sqrt{E_{\mathrm{d}} E_{\mathrm{q}}}}{E_{\mathrm{d}}+E_{\mathrm{q}}}$,

where $0 \leq C \leq 1$ is the purity (or amplitude correlation) factor. Thus, $C=1$ when $E_{\mathrm{d}}=E_{\mathrm{q}}$, and $C=0$ at the opposite extreme where the magnitude of one component is neglible compared to that of the other.

The time series of $E_{\mathrm{d}}, E_{\mathrm{q}}$ and $C$ for the interval $7500 \leq$ $t \leq 9500$ are shown in Fig. 4. Except for very short interludes where $P \approx+1$, the parity of the solution generally lies in the range $-1 \leq P \lesssim+0.5$ (see Fig. 1). Thus, the energy associated with the quadrupolar component of the magnetic field is generally smaller than that associated with the dipolar component. 


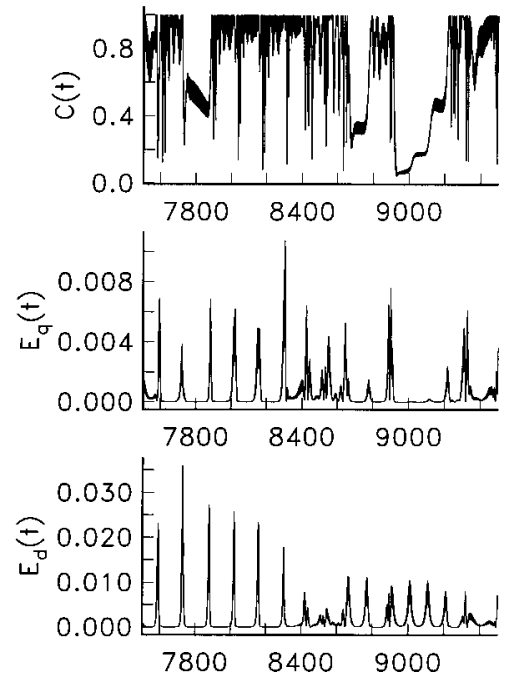

Fig. 4. The purity factor $C(t)$ (see text) and quadrupolar and dipolar magnetic energies for an interval of the calculation with $D=-400$, $\tau=10^{-3}$, cf. Fig. 1 .

During the interval $7900 \lesssim t \lesssim 8350$, there is a very strong correlation between $E_{\mathrm{d}}$ and $E_{\mathrm{q}}$. Throughout this regime, $C \approx 1$ except during short intervals which invariably follow a decay in magnetic energy. This interval corresponds to the first type of behaviour (in-out intermittency) described above.

\section{Are grand minima compatible with torsional oscillations?}

It is well established that, for small values of the low magnetic Prandtl number, the Malkus-Proctor mechanism can cause long-term modulations in magnetic energy (e.g. Tobias 1996, 1997; Moss \& Brooke 2000). If it is assumed that the shortterm oscillations correspond to the migratory sunspot cycle, then the extended periods of reduced activity may be identified as grand minima. However, there is an important difference between perturbations to the zeroth order rotation observed in our model and the torsional oscillations observed in the Sun. When $\tau \ll 1$, the kinetic energy of the perturbations of the model varies only on the longer timescale, thus torsional oscillations (ie. alternating migratory bands of slow and fast rotation) are not observed on the magnetic timescale.

To illustrate this point, it is convenient to consider the solution obtained with $\tau=0.1$ and $D=-300$, where oscillation periods $E_{\mathrm{m}}$ and $E_{\mathrm{k}}$ are similar and it is much easier to compare the respective butterfly diagrams. Contours of $B(x, t)$ and $v(x, t)$ computed just below the surface $(z=0.9)$ for this case are shown in Fig. 5a. We have restricted our attention to the low latitude region $-L / 2 \leq x \leq L / 2$, and subtracted a timeaveraged perturbation (measured at a latitude of approximately 25 degrees south, or $x \approx 5$ ) from $v(x, t)$, so that zones of faster (slower) than average rotation show up as positive (negative) contours (remembering that in this model the rotation has variation with depth but not latitude). Although the velocity perturbations clearly respond to the magnetic field with the same period ratio (1:2) observed in the Sun, the migration patterns do not follow the migration of the toroidal field, and have hardly any variation near the equator. When $\tau=1$ (lower panels) the correspondence between migration patterns is much more apparent, whereas for $\tau<0.1$ the torsional oscillations are much reduced.

A plausible explanation for the absence of torsional oscillations at small $\tau$ is that the viscous diffusion timescale is much longer than the magnetic, and hence the coupling between the velocity and magnetic fields is too weak for the former to respond to the Lorentz force before the polarity of the magnetic field reverses. If so, then increasing the magnetic Prandtl number should have a noticeable effect on the velocity perturbations. Contours of $B(x, t)$ and $v(x, t)$ computed for the same model discussed above, but with $\tau=1$, are shown in Fig. 5b. Although the toroidal field migrations are similar to those obtained with $\tau=0.1$, the velocity contours are now clearly divided into migratory bands of alternately fast and slow rotation, similar to the magnetic field oscillation patterns shown in Fig. 5.

These findings are consistent with those of Covas et al. (2001b), who used a spherical shell model with a solar-like zero order rotation law, and did not find torsional oscillations when $\tau \lesssim 0.4$. Also, using a rather different model, Küker et al. (1996) did find periodic variations in rotational velocity for $\tau \ll 1$, but only of the required amplitude for magnetic Prandtl numbers of $\tau \sim O(1)$ or greater. As the form of nonlinearity in the latter work was $\Lambda$-quenching and not the Malkus-Proctor effect, this suggests that having $\tau \gtrsim 1$ is a general requirement for reproducing the observed fluctuations in angular rotation. Where the magnetic Prandtl number becomes sufficiently low to produce the separation in timescales necessary for producing episodes of deep minima, the velocity perturbations can no longer respond on the magnetic timescale, thus the migration characteristic of torsional oscillations is lost.

We gain a particularly clear insight into this behaviour via Fourier analysis of the time series of the magnetic energy. In Fig. 6 we show the Fourier series of power vs frequency in the magnetic field for $D=-300,-400,-600,-800$ corresponding to the time series shown in Fig. 1. It can be seen that in all cases the main magnetic period at frequencies between 10 and 15 is completely detached from the low frequency power produced by the perturbations in the velocity field. These spectra are strongly bimodal, with essentially zero power separating the maxima. Thus on the timescale of the migratory magnetic wave the velocity field appears constant. The long term variations in velocity do show up in the broadening of the line due to the magnetic frequency but for each magnetic migratory wave the velocity profile appears static. It is also noticeable that the power at the low frequency is greater than the power of the magnetic cycle, this confirms the impression from the time series of Fig. 1 where the amplitude of the magnetic migratory oscillation is smaller than the amplitude caused by the velocity perturbations produced by the Malkus-Proctor effect.

\section{Conclusions}

Our aim in this investigation was to determine how the behaviour characteristic of stellar deep minima behaves with changing magnetic Prandtl number. We used a simple Cartesian 

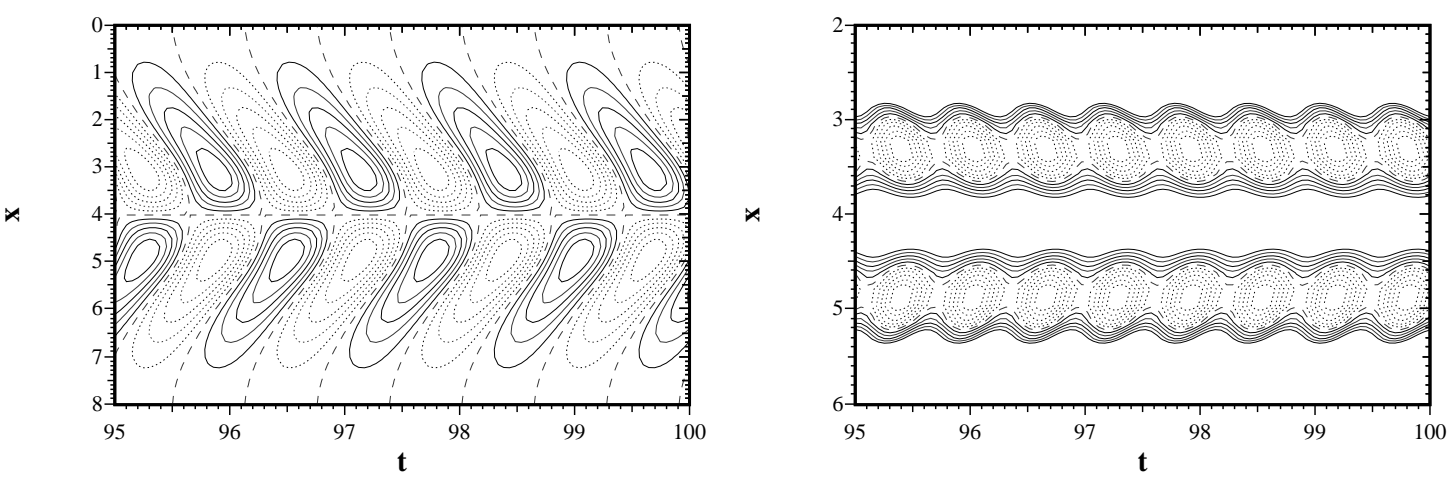

(a) $\tau=0.1$
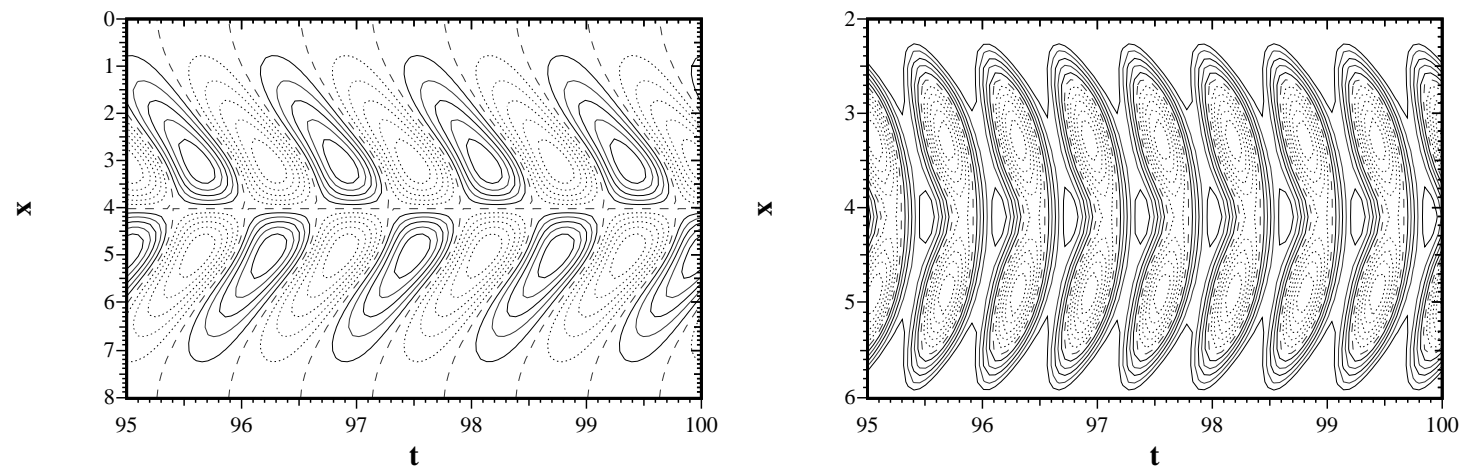

(b) $\tau=1$

Fig. 5. Contours of $B(x, t)$ (left) and $v(x, t)-\langle v(5, t)\rangle$ (right), i.e. butterfly diagrams, with $D=-300$ and given magnetic Prandtl numbers. In both cases, the migration patterns of the toroidal magnetic field are in approximate agreement with the behaviour observed during the 22 year Hale cycle of solar activity. However, torsional oscillations in the rotational velocity are only reproduced for magnetic Prandtl numbers of $\tau=O(1)$.

model with a single nonlinearity, namely the feedback of the velocity perturbations driven by the large scale magnetic field (Malkus-Proctor effect). We have elsewhere investigated the robustness of this model with respect to changes in both physics and geometry (Paper I). We found that the separation of length and time scales of the magnetic and viscous diffusion controls the dynamics and produces scalings related to $\tau$ by simple power law relationships over a range of magnetic Prandtl numbers. This confirms the conjecture of Tobias (1996) that such scalings exist, but our exponents are different from those suggested by Tobias. The dynamics of the small magnetic Prandtl number models appears quite rich, and we intend to return to this aspect in the future.

By investigating behaviour at very low magnetic Prandtl numbers we found that with this class of model the phenomenon of the deep minima is related to the behaviour of the self-killing dynamos described by Brummell et al. (1998), and Fuchs et al. (1999). The growth of the magnetic field produces, via the toroidal component of the Lorentz force, perturbations to the structure of the velocity field which produce an anti-dynamo configuration. The magnetic field then decays on the magnetic diffusion time. However, for small magnetic Prandtl numbers, the decay of the velocity perturbations is much slower, producing extended periods of very low magnetic energy. We confirmed this conjecture by examining the limiting case where the magnetic Prandtl number is zero and the velocity perturbations, once established, cannot decay. This was found to be a self-killing dynamo pure and simple, and the eventual frozen velocity field was found to depend on the initial conditions. We emphasize that our self-killing dynamo can be considered to be the limit of a series of models with increasingly intermittent behaviour, as the magnetic Prandtl number becomes very small. This behaviour is not found in models with quenching due to the local value of the field (e.g. alphaquenching) since they tend to reduce the effective dynamo parameters towards the critical values but cannot alter the model structure so as to produce an anti-dynamo configuration. Fuchs et al. (1999) also discussed a "self-generating" dynamo; we found no evidence for such a phenomenom with our model.

In the solar context we also note that our investigation highlights what we might call the Prandtl number dilemma. This states that if the magnetic Prandtl number is low enough to produce the separation of time scales characteristic of deep minima, then the velocity field cannot respond on the timescales of the magnetic migratory cycle. Thus one can have deep minima or torsional oscillations, the dilemma being that the or appears to be exclusive in this class of models. The importance of this point is such that we investigated it very carefully in Sect. 5. It has also been found in similar models in spherical geometry, using a similar nonlinear feedback. This dilemma is further 


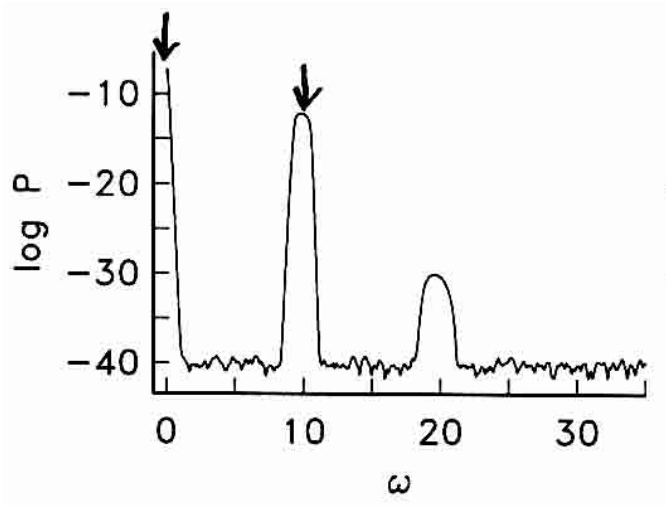

(a) $D=-300$

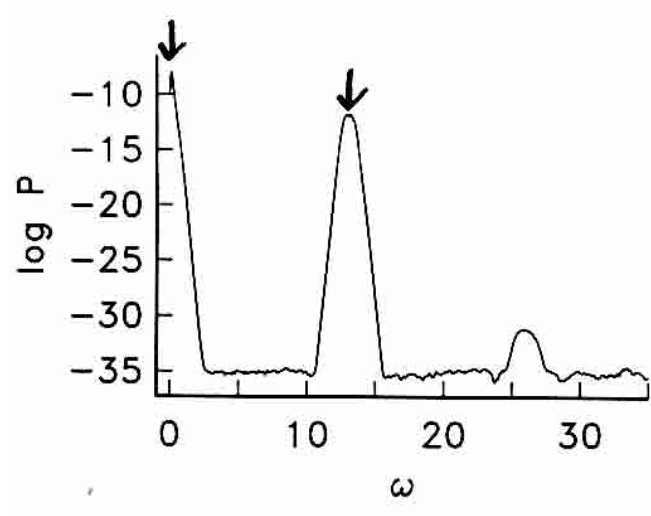

(c) $D=-600$

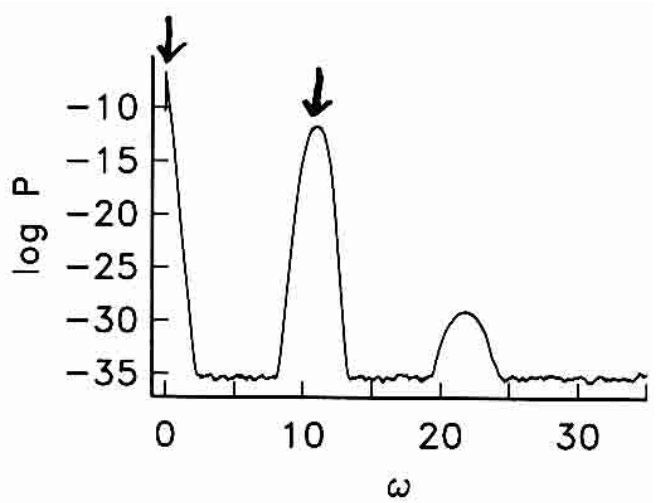

(b) $D=-400$

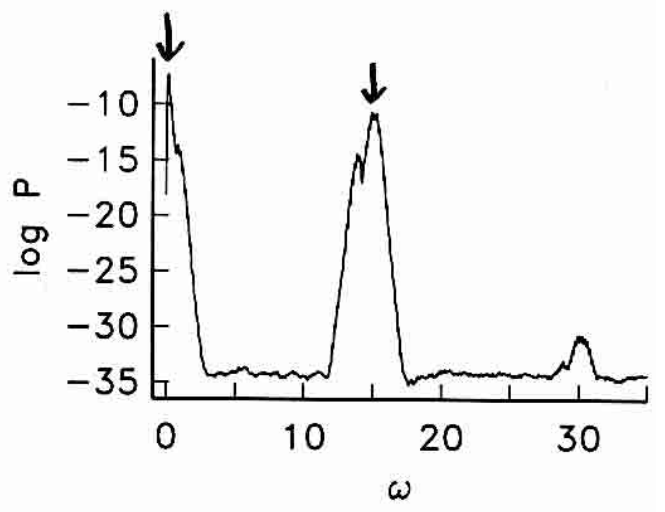

(d) $D=-800$

Fig. 6. Fourier series of power vs. frequency for the time series of magnetic energy shown in Fig. 1, i.e. $\tau=0.001, D=-300,-400,-600$, -800. Note the separation between the peak corresponding to the magnetic cycle and the low frequency power due to the Malkus-Proctor effect (indicated by arrows).

intensified by recent evidence from helioseismology that the torsional oscillations extend below the surface at least to a depth of $10 \%$ of the photospheric radius. Also, extensive investigations of dynamo models using the Malkus-Proctor feedback mechanism (e.g. Covas et al. 2000) show that with magnetic Prandtl numbers capable of producing torsional oscillations, they extend to the base of the convection zone (helioseismic inversions cannot yet give reliable information on oscillations at these depths). Thus they seem to be a primary phenomenon, not just a secondary surface effect. On the other hand, given the close observed relationship between the torsional oscillations and the magnetic field behaviour, it is perhaps hard to believe that they are not related, and the obvious mechanism is the Lorentz force (although in principle this could operate by, e.g., a quenching of the turbulent Reynolds stresses “ $\Lambda$-quenching", Küker et al. 1996). Covas et al. (2001b, c) have explored this issue more thoroughly.

Another generic problem with the low magnetic Prandt number models is that the grand minima are always as long as, if not much longer than the grand maxima. In contrast, the Sun appears to display extended intervals of more-or-less steady oscillatory behaviour, interspersed with grand minima. Given the analysis of the basic feedback mechanism presented in Sect. 4.1, the phenomenom of extended minima interspersed by grand maxima appears to be fundamental to simple models of the type discussed here.

Our overall conclusion is that, although a quite wide class of models can modulate the basic migratory magnetic cycle, they face severe difficulties on both theoretical and observational grounds. Currently we can only use observational checks on dynamo models for the sun. Here the "problem" is that we may be almost embarrassed by the large quantity of data available, but for other solar-type stars we have certainly have too few, although this is starting to change. (For example, a detection of surface differential rotation on a late-type dwarf star has been reported by Collier Cameron et al. 2002). Previous work (e.g. Moss \& Brooke 2000; Paper I) has shown how very sensitive the dynamo behaviour can be to self-driven small changes in the rotation profile. The sensitivity to small changes in the imposed background rotation law is less marked (e.g. Covas et al. 2001c), but larger changes do influence the overall behaviour of the dynamo (e.g. Moss \& Brooke 2000; Paper I). Thus care should be taken when extrapolating solar-type rotation laws to other stars. It is also important to assess the range of variation of behaviour due to the uncertainties in the profile deduced from observation. Currently this consideration only applies to the helioseismological data from the sun, but in future it may be more widely applicable to stellar dynamo models. 
A concrete manifestation of this sensitivity is that the power in the signal of the deep minima is considerably greater than the power in the migratory magnetic cycle. This is in sharp contrast to the sunspot cycle data over the last four centuries where the minima of the Schwabe cycle show very few sunpots even in periods where the amplitude of the cycle is greatest. Either some thresholding effect is masking the extent of the magnetic cycle variations, or else the solar deep minima exhibit dynamics very different from models with a Malkus-Proctor feedback mechanism (a survey of the literature describing such models shows this imbalance in power to be a general characteristic). The danger thus exists, that theoreticians can select those features of solar behaviour that are observed in their models and describe the behaviour as solar-like. This indicates that such claims should be verified by ensemble modelling to check the robustness of the behaviour in the models, and also by stating as precisely as possible exactly what features of the model are thought to be "solar-like". It is to be hoped that such precision will strengthen the link between theory and observation, to the benefit of both.

Acknowledgements. AP acknowledges support received under the Endowment Scholarship Scheme of the Faculty of Science and Engineering of the University of Manchester. We thank the referee, Axel Brandenburg, for a helpful report.

\section{References}

Ashwin, P., Covas, E., \& Tavakol, R. 1999, Nonlinearity, 9, 563

Baliunas, S. L., et al. 1995, ApJ, 438, 269

Brooke, J. M. 1997, Europhys. Lett., 37(3), 171

Brooke, J. M. 1997, Europhys. Lett., 38(2), 161 (Erratum)

Brooke, J. M., Pelt, J., Tavakol, R., \& Tworkowski, A. 1998, A\&A, 332,339
Brummell, N. H., Cattaneo, F., \& Tobias, S. M. 1998, Phys. Lett. A, 249, 437

Collier Cameron, A. C., Donati, J.-F., \& Semel, M. 2002, MNRAS, 330, 699

Covas, E., Tavakol, R., \& Moss, D. 2000, A\&A, 363, L13

Covas, E., Tavakol, R., Ashwin, P., Tworkowski, A., \& Brooke, J. M. 2001a, Chaos, 11, 404

Covas, E., Tavakol, R., \& Moss, D. 2001b, A\&A, 371, 718

Covas, E., Tavakol, R., Vorontsov, S., \& Moss, D. 2001c, A\&A, 375, L260

Eddy, J. A. 1976, Science, 192, 1189

Fuchs, H., Rädler, K.-H., \& Rheinhard, M. 1999, Astron. Nachr., 320 , 129

Howard, R., \& LaBonte, B. J. 1980, ApJ, 239, L33

Jennings, R. L., \& Weiss, N. O. 1991, MNRAS, 252, 249

Knobloch, E., \& Landsberg, A. S. 1996, MNRAS, 278, 294

Küker, M., Rüdiger, G., \& Pipin, V. V. 1996, A\&A, 312, 615

Malkus, W. V. R., \& Proctor, M. R. E. 1975, J. Fluid Mech., 67, 417

Moss, D., \& Brooke, J. 2000, MNRAS, 315, 521

Parker, E. N. 1993, ApJ, 408, 707

Phillips, A. D. 2001, Ph.D. Thesis, University of Manchester

Phillips, A., Brooke, J., \& Moss, D. 2002, A\&A, 392, 713 (Paper I)

Platt, N., Spiegel, E. A., \& Tresser, C. 1993a, Phys. Rev. Lett., 70, 279

Platt, N., Spiegel, E. A., \& Tresser, C. 1993b, Geophys. Astrophys. Fluid Dyn., 73, 147

Ribes, J. C., \& Nesme-Ribes, E. 1993, A\&A, 276, 549

Rüdiger, G. 1989, Differential Rotation and Stellar Convection (Springer Verlag Berlin; Berlin)

Schmitt, D., Schlüssler, M., \& Ferriz-Mas, A. 1996, A\&A, 311, L1

Sokoloff, D., \& Nesme-Ribes, E. 1994, A\&A, 288, 293

Tobias, S. M. 1996, A\&A, 307, L21

Tobias, S. M. 1997, A\&A, 322, 1007

Weiss, N. O., Cattaneo, F., \& Jones, C. A. 1984, Geophys. Astrophys. Fluid Dyn., 30, 305

White, O. R., Skumanich, A., Lean, J., Livingston, W. C., \& Keil, S. L. 1992, PASP, 104, 1139 